\title{
A Bidirectional Walk Method of Random Polygon Aggregate for Two-dimensional Concrete Model
}

\author{
Zheng CHEN ${ }^{1,2, a,{ }^{*}}$, Yu-Liu WEI ${ }^{1, b}$, Wei-Ying LIANG $^{1, \mathrm{c}}$ and Wo-Cheng \\ HUANG ${ }^{1, d}$
}

${ }^{1}$ Key Laboratory of Disaster Prevention and Structural Safety of China Ministry of Education, School
of Civil Engineering and Architecture, Guangxi University, Nanning 530004, China

${ }^{2}$ Department of Civil and Environmental Engineering, University of Alberta, Edmonton T6G 2W2, Canada

agxucz@qq.com, bgxuwyl@qq.com, cliangwy_kate@163.com, ’gxuhwc@qq.com

${ }^{*}$ Corresponding author

Keywords: Concrete, Random packing, Polygon aggregate, Bidirectional walk method.

\begin{abstract}
The distribution of aggregates is intensive at the bottom of concrete model but scarce at the top position when polygon aggregates are packed by traditional random walking algorithm. In order to simulate the randomness of aggregates distribution more effectively and realistically, a bidirectional walk method of random polygon aggregates is proposed to overcome the above shortage of the traditional method. Firstly, the method to judge whether or not polygon aggregates intersection exist during each aggregate packing cycle has been improved. Further, after all the aggregates move into the target domain, the reverse walk of aggregates is taken into account, which means the walk direction of the aggregates is changed to straight up from straight down. The main results obtained reveal that the distribution of polygon aggregates is more evenly in two-dimensional concrete model by the proposed method compared to the traditional one.
\end{abstract}

\section{Introduction}

Concrete is a kind of complex multi-phase composite material, but it is generally treated as a homogeneous material at the macro-levels in concrete mechanical properties and durability analysis. However, in meso-level, it is viewed as a kind of complex multiphase material consisting of aggregate, mortar matrix and the interfacial transition zone. An appropriate random aggregate model, considering gradation, content and shape of aggregates in concrete and so on, is the basis of successful simulation of meso-study of concrete.

The numerical simulation approach for concrete aggregate was proposed most early by Wittmann et al. [1]. They established an aggregate model that the random geometry of natural aggregates and composite structures can be simulated, and any particle size distribution can be taken into consideration. Then, by assuming aggregate particles to be perfect spheres, Schlangen et al. [2] and Liu et al. [3] both presented numerical model for numerical simulation of fracture of concrete materials and structures. In their model, aggregate structures are randomly generated according to the concrete mix. For boulder concrete, it is appropriate to assume aggregate to be round or sphere. But crushed stone concrete is frequently used in the production of construction, who has better mechanical properties than boulder concrete. And crushed stone concrete are required to establish polygon aggregate model to simulate. Thus Wang [4,5] established a packing algorithm for 2-D modeling of concrete aggregates with arbitrary shapes. Gao et al. [6] developed a numerical model to simulate the random aggregate structure. Conflict or overlap in the packing process were removed with conflict and overlap criteria based on an area index. Gong et al. [7] proposed an effective method for generating polygon aggregate, which can simulate aggregate distribution with any area percentage and any sides on arbitrary section. Gao et al. [8] proposed a method of generating convex polygon aggregate, considering the number of sides, shape and size simultaneously, which can ensure the convex characteristics of all the polygon aggregates. Therefore, there are many people who have been researching the method of generating random 
polygon aggregates. Then, based on random walk algorithm, Song et al. [9,10] proposed a numerical simulation method for round and polygon aggregate generation. This method can be applied to simulate aggregate movement in concrete pouring and mixing. Moreover, it can guarantee the aggregate generation successfully in one packing cycle, thus improve the efficiency of numerical simulation of concrete aggregate generation. But there is a disadvantage that it is difficult to control aggregate packing process, as a result, it is easy to make the aggregate distribute intensively at the bottom of concrete model but scarcely at the top, especially when aggregate content is low, there may even be blank space at the top of concrete model. That is, aggregate distribution is unreasonable in the concrete model.

The random walk method for polygon aggregate is ameliorated in this paper, and then a bidirectional walk method is proposed. By changing the walk direction of the aggregates to straight up from straight down, the aggregates are arranged evenly in concrete model. According to the comparison between the proposed method and traditional method, it can be proved that the proposed method is available to pack polygon aggregate continuously and evenly in the concrete model, and overcome the shortage of the traditional method, therefore it has an important significance on science and worthiness in practical application for analyzing concrete mechanical properties and durability.

\section{The Algorithm of Bidirectional Walk Method for Polygon Aggregate}

\section{Generate Polygon Aggregate Library}

Firstly, determining the size of aggregate grading interval, named $\left[d_{11}, d_{12}\right],\left[d_{21}, d_{22}\right], \ldots,\left[d_{n 1}, d_{n 2}\right]$, and the percentage of total aggregate content in each grading interval, named $\varphi_{1}, \varphi_{2}, \ldots, \varphi_{n}$. Then combining the total aggregate $\varphi$ and size of concrete model $W \times H$ to calculate the total area of polygon aggregate in each grading interval, named $A_{1}, A_{2}, \ldots, A_{n}$ as follows

$$
A_{n}=W \times H \times \varphi \times \varphi_{n}
$$

In every grading interval, the intermediate value of particle range are taken to be representative diameter of polygon aggregate, i.e., $\left(d_{\mathrm{n} 1}+d_{\mathrm{n} 2}\right) / 2$. For polygon aggregate, the half of representative diameter of aggregate means the distance from center point to vertices of aggregate. This method can not accurately consider the distance between vertices, but it is easier to determine the aggregate size. Based on representative diameter, the area of corresponding round aggregate can be calculated. Then the number of polygon aggregate particle in each grading range can be estimated by Eq. 2, and the value of $N_{\mathrm{n}}$ obtained through rounding-off is an integer.

$$
N_{n}=\frac{A_{n}}{\pi \cdot\left(\frac{d_{n 1}+d_{n 2}}{4}\right)^{2}}
$$

Then, as shown in Fig. 1, the random generation of polygon aggregate needs to determine the size of angle $\alpha_{1}, \alpha_{2} \ldots \ldots$ and the distance from center point to vertices of aggregate $r_{1}, r_{2} \ldots \ldots$ The angle can be randomly generated by

$$
\alpha_{1}^{\prime}=0 ; \quad \alpha_{i}^{\prime}=\alpha_{i-1}^{\prime}+45+45 \times \text { rand }
$$

where rand is random number between 0 and 1 , and for the polygon aggregate, the angle between horizontal and the line between the vertex and the center point is named $\alpha_{i}$, $i$ is the edge number of this polygon aggregate. Assuming that the particle size of polygon aggregate is the range $\left[d_{\min }, d_{\max }\right]$, the distance from center point to vertices of aggregate can be randomly generated by

$$
r_{i}=r_{\min }+\left(r_{\max }-r_{\min }\right) \times \text { rand }
$$

where rand is random number between 0 and $1, r_{\min }=d_{\min } / 2, r_{\max }=d_{\max } / 2$. To identify whether the 
aggregate generated is convex polygon or not, the inner function "convhull”of MATLAB software is applied in this process, which can return the convex hull of a set of points in 2-D or 3-D space. Then, the function "inpolygon" in MATLAB is used to determine whether the respective vertices are on the polygon boundary or not. If the aggregate generated before is concave polygon, then it needed to be regenerated.

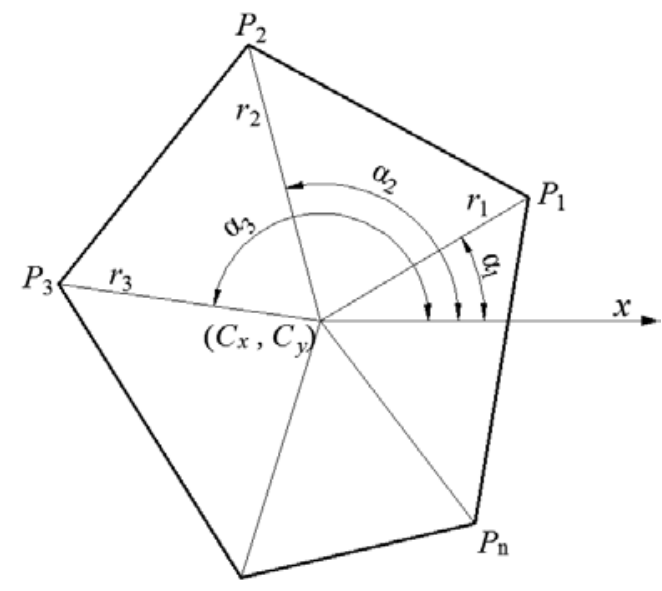

Fig.1 Polygon aggregate

Finally, according to the number of polygon aggregate particle in each grading range, the aggregates are generated one by one, and the angle and vertex coordinates are saved in polygon aggregate library.

\section{Randomly Packing and Falling Walking for Polygon Aggregate}

The order of packing is random. After polygon aggregate successively packed in waiting domain in concrete model, then based on the packing order, every polygon aggregate generated is compelled to walk straight down in vertical direction and left or right in horizontal direction, and revolve on its center, which can be expressed as Eq. 5. Note that when packing a polygon aggregate, it shall not overlap the aggregates which have already been in the waiting domain.

$$
c_{x, i}{ }^{\prime}=c_{x, i}+\operatorname{rand}_{1} \times r ; \quad c_{y, i}{ }^{\prime}=c_{y, i}-\operatorname{rand}_{2} \times r ; \quad \alpha_{i, j}{ }^{\prime}=\alpha_{i, j}+\operatorname{rand}_{3} \times \theta
$$

where $c_{x, i}, c_{y, i}$ and $c_{x, i}^{\prime}, c_{y, i}^{\prime}$ are the center coordinates of polygon aggregate before and after walking respectively, and $\alpha_{i, j}, \alpha_{i, j}^{\prime}$ are the angles of polygon aggregate before and after revolving respectively, $i$ is the numbering of polygon and $j$ is the numbering of vertex, and $\theta$ is the maximum revolving angle, rand ${ }_{1}$ is random number between -1 and 1, rand $_{2}$ and rand $_{3}$ are random number between 0 and $1, r$ is the walking step. According to the $c_{x, i}^{\prime}, c_{y, i}^{\prime}$ and $\alpha_{i, j}^{\prime}$, the vertex coordinates of polygon aggregate after walking can be calculated by

$$
x_{i}=c_{x, i}{ }^{\prime}+r_{i} \times \cos \alpha_{i}^{\prime} ; \quad y_{i}=c_{y, i}{ }^{\prime}+r_{i} \times \sin \alpha_{i}^{\prime}
$$

Each polygon aggregate should be judged whether or not its movement is effective after walking.

Firstly, determining whether or not the aggregate has intersection with boundary after walking. Due to the aggregate walking straight down in vertical direction and left or right in horizontal direction, there is a need to guarantee that the vertexes of polygon aggregate will not exceed the left, right and bottom boundary of the target domain, which can be expressed as

$$
0+C \leq x_{i, j} \leq 100-\mathrm{C} ; y_{i, j} \geq 0+C
$$

where $C$ is distance parameter between aggregate and boundary, $x_{i, j}$ and $y_{i, j}$ are the coordinates of 
polygon vertex.

Then, judging whether or not the polygon aggregate after walking intersects with the other aggregates. Because there are many situation of polygon intersection, it is complex to determine this. Based on the discussion of Song [10], the inner function "polybool”of MATLAB software is selected in this process and make it easier. The function "polybool" performs the polygon set operation about intersection, union, subtraction and exclusive or. Thus the intersection of two aggregates can be successively obtained by this function. And if the return numbers of intersection are all empty array, it means that the aggregate after walking doesn't intersect with the other. Obviously, the function "polybool" can be used to determine whether or not there is polygon aggregate intersection easier. However, this approach would make the space between aggregates too intensive, as a result, it is only suitable for high content of aggregate packing. When it comes to the low aggregate content, the other method is proposed to determine that any two aggregates have no intersection, as shown in the following equation

$$
c_{i} c_{k}>D \times\left(r_{i, \max }+r_{k, \max }\right)
$$

where $D$ is the distance parameter between aggregates, $C_{i} C_{k}$ is the distance between centers of any two polygon aggregate, $r_{i, \max }$ and $r_{k, \max }$ are the maximum distance between center and vertex of any two polygon aggregate.

If the aggregates after walking satisfy all of the conditions mentioned above, this indicates that this walking is effective, and then the coordinates of aggregate after walking should be saved so that the next walking can continue. If any of the conditions is not satisfied, then discard the results of walking to keep aggregate in original position.

\section{Randomly Reverse Walking for Polygon Aggregate}

When it comes to randomly reverse walking, all aggregates will be walking in reverse direction from the last one to the first one. Each polygon aggregate generated is compelled to walk straight down to straight up in vertical direction and left or right in horizontal direction, and revolve on its center, which can be expressed as

$$
c_{x, i}{ }^{\prime}=c_{x, i}+\operatorname{rand}_{1} \times r ; \quad c_{y, i}{ }^{\prime}=c_{y, i}+\operatorname{rand}_{2} \times r ; \quad \alpha_{i, j}^{\prime}=\alpha_{i, j}+\operatorname{rand}_{3} \times \theta
$$

According to the $c_{x, i}^{\prime}, c_{y, i}^{\prime}$ and $\alpha_{i, j}^{\prime}$, the vertex coordinates of polygon aggregate after walking can be calculated by Eq. 6 .

Each polygon aggregate should be judged whether or not its movement is effective after walking.

Firstly, determining whether or not the aggregate has intersection with boundary after walking. Due to the aggregate walking straight down to straight up in vertical direction and left or right in horizontal direction, there is a need to guarantee that the vertexes of polygon aggregate never exceed the left, right and upper boundary of the target domain, which can be expressed as

$$
0+C \leq x_{i, j} \leq 100-C ; y_{i, j} \leq 100-C
$$

where $C$ is distance parameter between aggregate and boundary, ${ }^{x_{i, j}}$ and ${ }^{y_{i, j}}$ are the coordinates of polygon vertex.

Then, the method concerning judging whether or not the polygon aggregate after walking intersects with the other aggregate is similar to that mentioned in falling walking.

If the aggregates after walking satisfy all of the conditions mentioned above, this means that this walking is effective, and then the coordinates of aggregate after walking should be saved so that the next walking can continue. If any of the conditions is not satisfied, then discard the results of walking to keep aggregate in original position. According to the PDE tool in MATLAB, we can check every aggregate's position after walking. If there is any aggregate not be in an ideal position, we can make it reversely walking in a little distance repeatedly till all aggregates are packed evenly in the model. 


\section{Analysis for Aggregate Distribution in Concrete Domain}

The two-dimensional concrete domain is divided into several aggregate distribution areas, and then the polygon aggregate content in each area can be calculated. Furthermore, to evaluate the quality of random packing for polygon aggregate, the standard deviation and variation coefficient of aggregate content should be calculated.

\section{Analysis of Example}

\section{Generate Polygon Aggregate Library}

In this example, grading interval of the polygon aggregate were $5-10 \mathrm{~mm}, 10-15 \mathrm{~mm}, 15-20 \mathrm{~mm}$ and $20-25 \mathrm{~mm}$, respectively. The aggregate content of this four grading interval all accounted for $25 \%$ of total aggregate. The target contents of aggregate packing were $30 \%, 40 \%$ and $50 \%$, respectively. The size of waiting domain in concrete model was $100 \mathrm{~mm} \times 25 \mathrm{~mm}$ and the size of target domain was $100 \mathrm{~mm} \times 100 \mathrm{~mm}$, as shown in Fig. 2. According to the shape and gradation of aggregate, the representative diameter of aggregate was determined. As for each grading interval, the intermediate value of it was taken to be representative diameter of polygon aggregate, i.e., $7.5 \mathrm{~mm}, 12.5 \mathrm{~mm}, 17.5 \mathrm{~mm}$ and $22.5 \mathrm{~mm}$, respectively. For the polygon aggregate, the distance from center point to vertices of aggregate particle were $3.75 \mathrm{~mm}, 6.25 \mathrm{~mm}, 8.75 \mathrm{~mm}$ and $11.25 \mathrm{~mm}$ respectively. And then combined with the content of aggregate packing and the size of target domain, the polygon aggregate library was generated. After aggregate generated, there were 40, 50 and 60 aggregates in the model with aggregate content of $30 \%, 40 \%$ and $50 \%$ respectively.

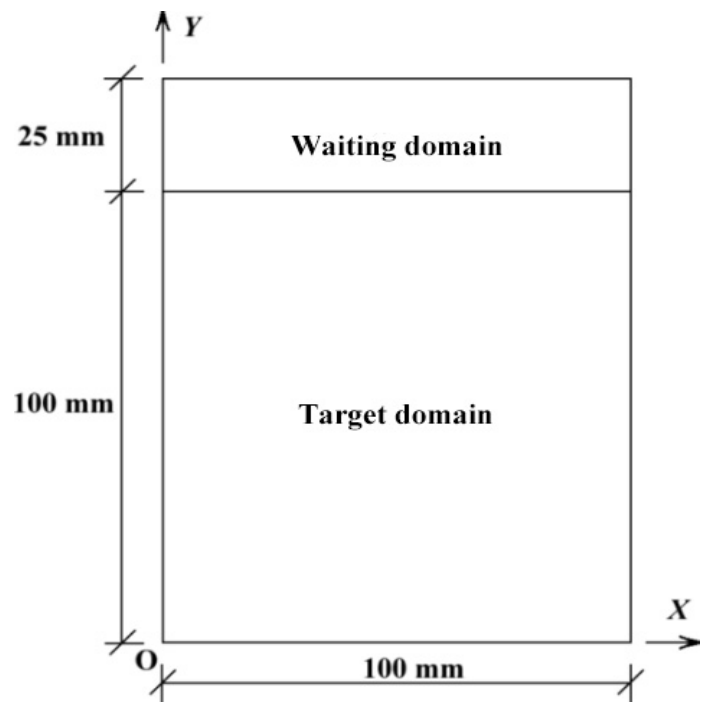

Fig. 2 The waiting and target domain in concrete model

\section{Randomly Packing and Falling Walking for Polygon Aggregate}

The packing order of polygon aggregate was random. After polygon aggregate successively packed in waiting domain, based on the packing order, every polygon aggregate generated was compelled to walk straight down in vertical direction and left or right in horizontal direction, and revolved on its center. For the purpose of comparison, the value of $C$ and $D$ was 1.1 in this example, and the value of walking step was $10 \mathrm{~mm}$. The walking times of aggregates during each packing was 20 . In the final packing, aggregates were compelled to stop walking when they all moved into target domain. As can be seen from Fig. 3, after the traditional falling walking, the concrete models with aggregate content of $30 \%, 40 \%$ and $50 \%$ respectively were generated, and then the two-dimensional concrete domain was divided into four aggregate distribution areas. Furthermore, the polygon aggregate content of each area was calculated and the standard deviation and variation coefficient of aggregate content were analyzed, as shown in Table 1. 


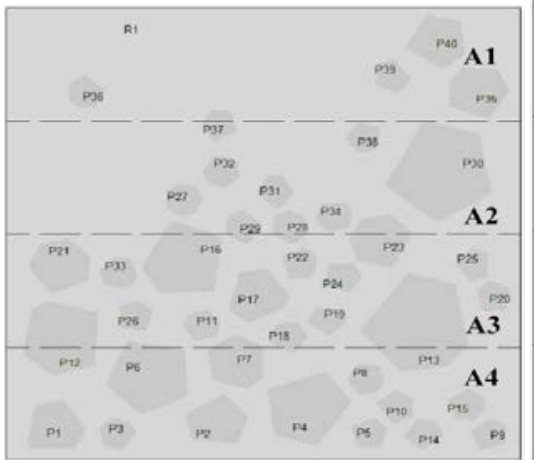

(a) $30 \%$

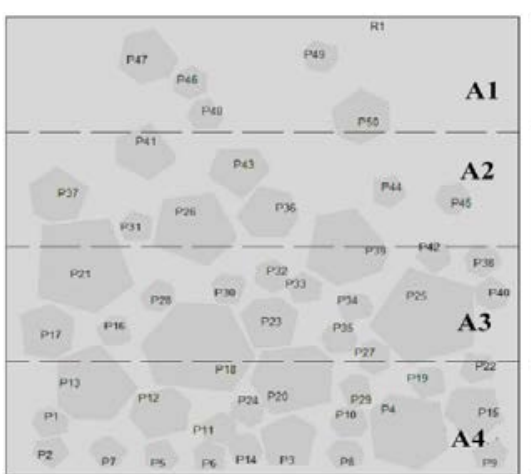

(b) $40 \%$

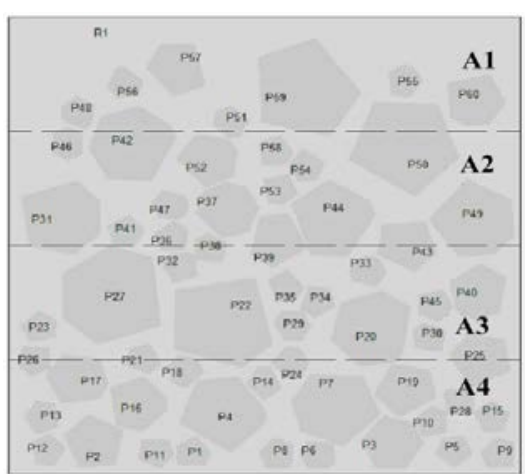

(c) $50 \%$

Fig. 3 The concrete models with different polygon aggregate content based on traditional walk method

Table 1 The polygon aggregate distribution based on traditional walk method

\begin{tabular}{|c|c|c|c|c|c|c|}
\hline $\begin{array}{c}\text { Target } \\
\text { aggregate } \\
\text { content }\end{array}$ & $\begin{array}{c}\text { Aggregate } \\
\text { content of } \\
\text { A1 }\end{array}$ & $\begin{array}{c}\text { Aggregate } \\
\text { content of } \\
\text { A2 }\end{array}$ & $\begin{array}{c}\text { Aggregate } \\
\text { content of } \\
\text { A3 }\end{array}$ & $\begin{array}{c}\text { Aggregate } \\
\text { content of } \\
\text { A4 }\end{array}$ & $\begin{array}{c}\text { The } \\
\text { standard } \\
\text { deviation }\end{array}$ & $\begin{array}{c}\text { The } \\
\text { variation } \\
\text { coefficient }\end{array}$ \\
\hline $30 \%$ & $10.75 \%$ & $24.04 \%$ & $45.21 \%$ & $39.60 \%$ & $13.50 \%$ & 0.45 \\
\hline $40 \%$ & $11.77 \%$ & $32.35 \%$ & $59.71 \%$ & $55.94 \%$ & $19.40 \%$ & 0.48 \\
\hline $50 \%$ & $30.74 \%$ & $57.04 \%$ & $56.53 \%$ & $56.26 \%$ & $11.20 \%$ & 0.22 \\
\hline
\end{tabular}

\section{Randomly Reverse Walking for Polygon Aggregate}

When it comes to randomly reverse walking, all aggregates were compelled to walk in reverse direction from the last one to the first one. Based on the bidirectional walk method, the concrete models with polygon aggregate content of 30\%, $40 \%$ and $50 \%$ differently were generated, as shown in Fig. 4. Moreover, the polygon aggregate content of four areas and the standard deviations and variation coefficients of aggregate content were given in Table 2.

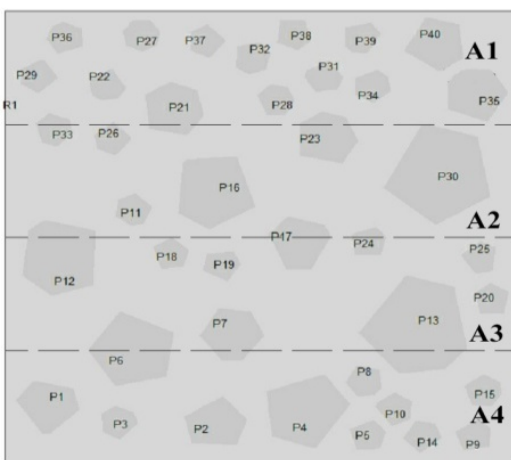

(a) $30 \%$

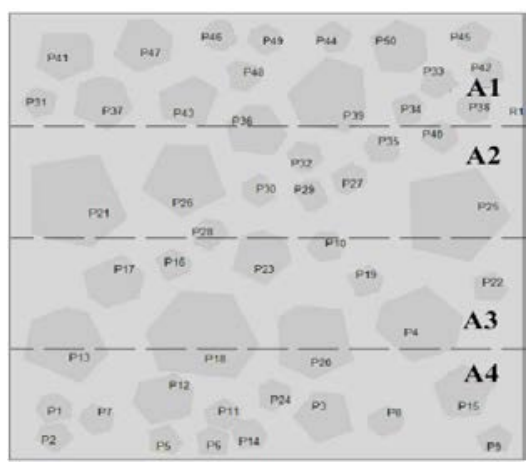

(b) $40 \%$

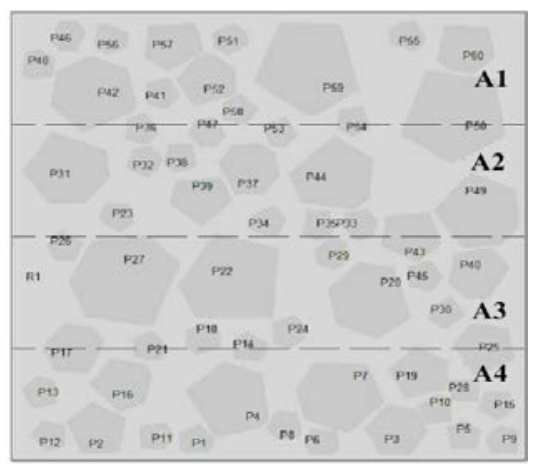

(c) $50 \%$

Fig. 4 The concrete models with different polygon aggregate content based on bidirectional walk method

Table 2 The polygon aggregate distribution based on bidirectional walk method

\begin{tabular}{|c|c|c|c|c|c|c|}
\hline $\begin{array}{c}\text { Target } \\
\text { aggregate } \\
\text { content }\end{array}$ & $\begin{array}{c}\text { Aggregate } \\
\text { content of } \\
\text { A1 }\end{array}$ & $\begin{array}{c}\text { Aggregate } \\
\text { content of } \\
\text { A2 }\end{array}$ & $\begin{array}{c}\text { Aggregate } \\
\text { content of } \\
\text { A3 }\end{array}$ & $\begin{array}{c}\text { Aggregate } \\
\text { content of } \\
\text { A4 }\end{array}$ & $\begin{array}{c}\text { The } \\
\text { standard } \\
\text { deviation }\end{array}$ & $\begin{array}{c}\text { The } \\
\text { variation } \\
\text { coefficient }\end{array}$ \\
\hline $30 \%$ & $27.29 \%$ & $29.09 \%$ & $31.71 \%$ & $30.70 \%$ & $1.64 \%$ & 0.05 \\
\hline $40 \%$ & $41.77 \%$ & $40.64 \%$ & $41.10 \%$ & $36.44 \%$ & $2.09 \%$ & 0.05 \\
\hline $50 \%$ & $49.79 \%$ & $48.33 \%$ & $52.11 \%$ & $50.31 \%$ & $1.35 \%$ & 0.03 \\
\hline
\end{tabular}


Compared Fig. 3 and Fig. 4, after traditional walking, aggregates are distributed intensively at the bottom but scarcely at the top of the target domain in concrete model, especially when the aggregate content is low, there may even be blank space at the top of the target domain. Through randomly reverse walking, aggregates are distributed in target domain evenly. Therefore, the bidirectional walk method surmounts the defects belong to traditional walk method.

Compared Table 1 and Table 2, the standard deviations and variation coefficients of aggregate content after traditional walking are much higher than that by bidirectional walking, and the variation coefficient of one group is up to 0.48 . However, the variation coefficient of aggregate content can be controlled within 0.05 on the basis of utilizing bidirectional walk method. This can prove that the bidirectional walk method is capable of ensuring the aggregates to distribute evenly in target domain.

\section{Conclusions}

This paper investigates numerically the random distribution of polygon aggregates. A bidirectional walk method of random polygon aggregates for two-dimensional concrete model is proposed. Based on the traditional walk method of aggregates, some improvements have been finished. Firstly, the method to judge whether or not polygon aggregates intersection exist during each aggregate packing cycle has been improved. Further, a reverse walk of aggregates has been taken into account to improve evenness of polygon aggregates in model.

Several case study performed at different aggregate contents have been taken as the examples, the method proposed is adopted to simulate the process of polygon aggregates pack. The numerical results show that the variation coefficient of aggregate content can be controlled within 0.05 on the basis of utilizing bidirectional walk method. This means that the aggregate content in each analysis area is approximate to our target aggregate content and the difference can be acceptable.

In the course of this analysis, we can find that the proposed method has surmounted the shortage concerning intensive aggregates distribution at the bottom of concrete model but scarce aggregates distribution at the top position of traditional method. Such an improvement reveals the value of the method presented, which is that it helps us to enable the aggregates to distribute more evenly in concrete model while doing the corresponding numerical research, simultaneously, propels our numerical simulation to be approximate to reality situation.

\section{Acknowledgements}

This work was sponsored by the National Natural Science Foundation of China (51208120 \& 51468004), the Projects of Guangxi Natural Science Foundation (2014GXNSFAA118309, 2015GXNSFBB139011 \& 2012GXNSFEA053002-2) and Guangxi Technological Development Projects (1598009-10).

\section{References}

[1] Wittmann F, Roelfstra P, Sadouki H. Simulation and Analysis of Composite Structures, J. Materials Science and Engineering. 68(2) (1985) 239-248.

[2] Schlangen E, van Mier J G M. Simple lattice model for numerical simulation of fracture of concrete materials and structures, J. Materials and Structures. 25 (1992) 534-542.

[3] Guang-Ting LIU, Zong-Min WANG. Numerical simulation study of fracture of concrete materials using random aggregate model, J. Journal of Tsinghua University (Sci \&Tech). 36(1) (1996) 84-89. (In Chinese)

[4] Z.M. Wang, A.K.H. Kwan, H.C. Chan. Mesoscopic study of concrete I: Generation of random aggregate structure and finite element mesh, J. Computers and Structures. 70 (1999) 533-544.

[5] Zong-Min WANG. Mesoscopic study of crack growth and composite behavior of concrete, D. 
Beijing, Tsinghua University. 1996. (In Chinese)

[6] Zheng-Guo GAO, Guang-Ting LIU. Two-dimensional random aggregate structure for concrete, J. Journal of Tsinghua University (Sci \&Tech). 43(5) (2003) 710-714. (In Chinese)

[7] Zheng-Lu GONG, Jun-Tao YANG. Random aggregate structure and size effect for concrete, J. Low Temperature Architecture Technology. 33(6) (2011) 11-13. (In Chinese)

[8] Qiao-Hong GAO, Zhen-Qun GUAN, Yuan-Xian GU et al. Automatic generation of finite element model for concrete aggregate, J. Journal of Dalian University of Technology. 46(5) (2006) 641-646. (In Chinese)

[9] Xiao-Gang SONG, Zhi-Chun YANG. A new method to simulate round concrete aggregate generation, J. Engineering Mechanics. 27(1) (2010)154-159, 172. (In Chinese)

[10] Xiao-Gang SONG, Zhi-Chun YANG. Random walking algorithm for polygonal concrete aggregate packing, J. Chinese Journal of Applied Mechanics. 26(4) (2009) 808-811,843. (In Chinese) 